Cahiers $d u$ MONDE RUSSE

\section{Cahiers du monde russe}

Russie - Empire russe - Union soviétique et États indépendants

61/3-4 | 2020

Écritures visuelles, sonores et textuelles de la justice

\title{
Fabian THUNEMANN, Verschwörungsdenken und Machtkalkül. Herrschaft in Russland, 1866-1953
}

\section{Gábor T. Rittersporn}

\section{OpenEdition}

\section{Journals}

\section{Édition électronique}

URL : https://journals.openedition.org/monderusse/12148

DOI : 10.4000/monderusse. 12148

ISSN : $1777-5388$

\section{Éditeur}

Éditions de l'EHESS

\section{Édition imprimée}

Date de publication : 1 juillet 2020

Pagination : 530-534

ISBN : 978-2-7132-2832-2

ISSN : $1252-6576$

Référence électronique

Gábor T. Rittersporn, «Fabian THUNEMANN, Verschwörungsdenken und Machtkalkül. Herrschaft in Russland, 1866-1953», Cahiers du monde russe [En ligne], 61/3-4 | 2020, mis en ligne le 01 juillet 2020 consulté le 05 septembre 2022. URL : http://journals.openedition.org/monderusse/12148 ; DOI https://doi.org/10.4000/monderusse. 12148 
Fabian THUNEMANN

\section{Verschwörungsdenken und Machtkalkül}

Herrschaft in Russland, 1866-1953

Berlin : De Gruyter Oldenburg (Ordnungssysteme. Studien zur Ideengeschichte der Neuzeit, vol. 53), 2019, 270 p.

On découvre de nos jours des conspirations et des fantasmes conspirationnistes à propos de tout ce qui se passe, ou presque. De tout ce qui se passe, ou ne se passe pas. Le phénomène laisse rêveur l'historien. Il est souvent confronté à l'interprétation, que font les acteurs qu'il observe, de rapports sociaux enchevêtrés et de processus politiques complexes en termes d'intrigues, de conspirateurs qui agiraient surtout dans les allées du pouvoir. L'historien sait par expérience que l'imagerie de complots omniprésents caractérise des régimes qui ont beaucoup de 
choses à cacher, avant tout des régimes qui institutionnalisent l'obscurité des lieux où se décide le sort des citoyens et la dissimulation des personnes qui semblent prendre des décisions de grande portée. Cette vision est alimentée par le vécu du public et par les informations que celui-ci tient des media qui suggèrent parfois que les pires méfaits peuvent être attribués aux élites.

Notons, avant d'oser nous aventurer plus loin, que l'intéressant livre de Fabian Thunemann risque de contribuer à la perplexité du chercheur. Thunemann explore l'imagerie de la conspiration en Russie et des figures de pensée conspirationnistes qui apparaissent dans les milieux gouvernementaux à partir des dernières décennies de l'ancien régime et perdurent au-delà, influencent leurs initiatives et peuvent motiver l'utilisation politique des menaces qu'elles sous-entendent. La perplexité du lecteur vient de l'actualité du sujet, qu'il est difficile d'ignorer, et du malentendu qu'elle est susceptible d'engendrer. Car Thunemann n'a pas l'intention de traiter des problèmes du temps présent. Malgré tout, son travail aboutit à une phénoménologie historique qui prête à confusion. Autant dire que ses analyses produisent des résultats dont l'importance transcende ses recherches.

À partir du milieu du XIX ${ }^{\mathrm{e}}$ siècle, l'autocratie des tsars dut affronter des troubles fomentés par des groupes de révolutionnaires qui voulaient renverser le régime. Souvent, les membres de ces groupes planifiaient des attentats contre les représentants du pouvoir. Ils en ont tué un certain nombre. La personne du tsar était une cible privilégiée des conjurés aussi bien que des terroristes qui n'étaient pas affiliés à un complot. Le coup le plus spectaculaire d'une conspiration fut l'assassinat d'Alexandre II en 1881. Le régime a répondu énergiquement. Malgré les réformes de la justice, qui instituaient des cours largement indépendantes du gouvernement, la menace terroriste a donné lieu à une pratique pénale d'exception. Dans une certaine mesure, le gouvernement réagissait plus contre des conjurations potentielles que contre celles qu'il avait toutes les raisons de neutraliser. La simple possibilité de complots meurtriers motivait des actions en fait dirigées contre un imaginaire, qui a commencé à devenir une réalité aussi tangible que les attentats qui coûtaient la vie de politiciens éminents.

Les bolcheviks ont emboîté le pas. Conspirateurs chevronnés eux-mêmes, ils étaient enclins à chercher partout des groupes clandestins. Les assassinats d'un petit nombre de fonctionnaires illustres et des tentatives d'assassinat, notamment celle dont Lenin a failli être victime, ont intensifié la crainte des conspirations. Les bolcheviks voulaient dépister des complots à tout prix et croyaient en découvrir, même quand les contre-révolutionnaires agissaient manifestement seuls, quand aucun péril ne se profilait à l'horizon, quand tel ou tel autre groupe n'avait rien de clandestin ni aucune intention de s'attaquer au régime, même quand les associations liquidées étaient tout à fait imaginaires. Quelques rares conjurations et la lutte des Diadochi qui a suivi la mort de Lenin ont renforcé les craintes de la clique en place au sommet du régime. Celle-ci agissait de plus en plus comme une fomentatrice de complots, même aux yeux de cadres dirigeants, dont un certain nombre étaient accusés d'intriguer contre la direction suprême quand ils se risquaient à émettre des doutes sur la politique dictée par les sommités de l'État-Parti. 
S'il y avait de moins en moins de raison de dépister des cabales au sein de l'appareil vers le milieu de la décennie d'avant-guerre, l'assassinat, en 1934, de Sergej M. Kirov, secrétaire du Parti de Leningrad et membre du Politbjuro, avait tout pour évoquer le spectre des complots terroristes. L'attentat avait manifestement été perpétré par un individu isolé. Il fut néanmoins attribué à un groupe dont le procès à huis clos servit de prétexte pour inculper Grigorii E. Zinov'ev et Lev B. Kamenev, politiciens déchus et participants illustres aux conflits des factions rivales dans les années 1920. Après tout, ils étaient des conspirateurs potentiels. Les rapports policiers sur l'ambiance parmi les masses ont révélé une certaine sympathie pour l'assassin, que d'aucuns comparaient aux révolutionnaires du $\mathrm{XIX}^{\mathrm{e}}$ siècle. Ceci suffisait pour accroître la peur pour leur vie des dirigeants les plus éminents de l'État-Parti, Stalin en premier lieu.

Thunemann ne s'attarde pas sur les petits groupes secrets qui étaient susceptibles de contribuer à alimenter l'anxiété ambiante et dont les adhérents rêvaient d'un changement de régime, le plus souvent selon un modèle bolchevik qu'ils jugeaient authentique, à la différence de celui représenté par l'État-Parti qu'ils qualifiaient de dégénéré et condamnaient. Peu importe. Il met en évidence des craintes complètement détachées de ce qu'on pouvait observer dans la société soviétique, craintes qui ont fini par vivre une vie autonome. Moins il y avait de véritables complots, plus les dirigeants supposaient qu'ils étaient soigneusement masqués et échappaient à la vigilance de la police secrète.

Selon l'auteur, Stalin est devenu prisonnier des soupçons engendrés par le danger potentiel de cabales qu'il croyait déceler même dans son entourage immédiat après la guerre. Il entreprenait des manœuvres byzantines pour conjurer la menace. Stalin est le principal personnage du livre, à telle enseigne que son patronyme n'apparaît pas dans l'index des noms propres : il faudrait citer tellement de pages que le registre perdrait tout son sens. On exagère à peine en disant que le lecteur risque d'être gagné par l'impression que la principale occupation du dictateur était de lutter contre toutes sortes de complots imaginaires. En parlant de l'ancien régime, Thunemann place le récit dans le contexte d'événements comme la libération des serfs, la réforme de la justice et de l'administration locale ou la guerre russo-japonaise. Les grandes entreprises du régime soviétique comme l'offensive contre le monde rural, la création quasiment ex nihilo d'une industrie, la Grande Terreur ou la Seconde Guerre mondiale ne sont même pas des bruits de fond. Le récit tourne autour des menaces fantasmées et décrit des intrigues au sommet de l'État-Parti, surtout celles de Stalin qui voit son autorité et sa vie constamment menacées.

En outre, on sait de sa correspondance avec ses lieutenants que Stalin n'était pas tout à fait convaincu que ses sujets obéissent à ses ordres, même quand il s'agissait de camarades de longue date évoluant dans son environnement immédiat. Cette fois, ce sont l'économie nationale ou le fonctionnement de l'administration qui étaient en jeu. Le leader s'intéressait virtuellement à tout ce qui concernait l'industrie lourde et quelques grands projets ; il se mêlait des détails de la planification d'une économie qui, pourtant, échappait aux desseins des planificateurs, y compris le plus puissant d'entre eux ; il mettait même en forme des directives 
du Politbjuro, allant jusqu'à corriger leur ponctuation. Il suivait de près tout ce qui touchait à l'armée et au complexe militaro-industriel ainsi qu'aux opérations pendant la guerre. Il ne négligeait pas le cours de la terreur déchaînée en 1937 et 1938 et étudiait les confessions d'officiels arrêtés, même celles de sous-fifres, alors qu'il savait bien qu'elles étaient le plus souvent obtenues par la torture, qu'il avait lui-même autorisée au début de la campagne. Manifestement, à ses yeux, pouvoir ne signifiait pas simplement sécurité de sa personne et intangibilité de son statut de Dirigeant suprême, cela signifiait aussi obéissance à ses ordres.

Il n'ignorait pas que rien n'était moins sûr qu'ils fussent respectés. N'était-il pas prêt à se plaindre à la tribune d'un congrès du Parti que l'administration de l'agriculture voyait sa mission principale dans la dissimulation de l'état des affaires de sa branche? Ne devait-il pas constater que l'épuration meurtrière des appareils de l'État-Parti ne parvenait pas à garantir la soumission inconditionnelle des cadres de tous rangs aux directives du sommet, de même qu'elle ne décourageait pas la reconstitution de leurs cliques et leurs pratiques dilatoires qui avaient motivé les accusations de complot et de sabotage qui avaient conduit à l'élimination de leurs prédécesseurs?

Thunemann reconnaît l'importance des menées des apparatchiks et de la menace hypothétique de tendances centrifuges, quelle que soit leur ampleur. Mais son propos n'est pas là. Il veut montrer, qu'indépendamment de la portée des machinations dans les appareils et malgré le fait que les conspirations - que des esprits formés par l'expérience de leurs propres complots subodorent —, étaient fantasmées, les dangers crédibles qu'elles suggéraient étaient devenus des faits incontestables et motivaient des réactions contre des chimères. Il y parvient. Si bien que son travail pose dans le contexte soviétique la question des relations entre les faits durs que l'historien saisit dans l'univers d'une société et l'imaginaire politique qui entretient des rapports éloignés avec ce qu'on peut prendre pour le réel. Aussi, il démontre, qu'indépendamment de tout ce que les dirigeants pouvaient croire, c'est moins la réalisation de leurs projets et le respect de leurs ordres qui les motivaient que leur monopole de les formuler.

À certains égards, l'analyse de Thunemann touche à la problématique de Cornelius Castoriadis, qui décrit la constitution imaginaire de la société. La société de notre auteur est un milieu restreint, institué par une imagerie simpliste qui ne saisit même pas les vagues contours du monde que les acteurs croient déchiffrer. On peut arguer que le penchant de comprendre les rapports sociaux et politiques en termes de conspirations omniprésentes est un trait caractéristique de toute la société soviétique, en particulier à l'époque qui intéresse Thunemann. Malgré tout, c'est l'expérience quotidienne du sujet ordinaire qui fonde les représentations du quidam, alors que celles des dirigeants viennent de la potentialité d'événements qui n'ont guère de chances de se produire.

Il n'est pas simple de rendre compte de cet ouvrage, qui est un véritable tour de force. Il est complexe, dense et fondé sur une multitude de détails de l'histoire russe et soviétique, qui sont rarement associés entre eux et en partie peu connus. Il propose une réflexion sophistiquée sur le politique, sur le pouvoir et sur ses usages. 
Les savants qui explorent les régimes où les tenants et les aboutissants des processus politiques sont peu clairs ont tout intérêt à feuilleter l'ouvrage de Thunemann. Il en va de même pour les adeptes des théories de la conspiration. Ceux-ci et ceux-là ont certainement relevé que, parfois, les dirigeants attribuent des événements qu'ils peinent à comprendre aux machinations de meneurs bien masqués ou aux manigances de puissances étrangères. Les chercheurs et les amateurs de l'idée de la main cachée peuvent aussi méditer sur le fait de savoir si les plus éminents des citoyens éminents sont davantage motivés par la réalisation des programmes qu'ils annoncent que par leur volonté d'appartenir à l'élite de l'élite dirigeante. En tout cas, les scientifiques et le public en général pourraient se poser la question de savoir si, et à quel point, les stratégies politiques qu'ils observent et subissent sont des réactions à des faits empiriquement vérifiables et dans quelle mesure ces réactions sont fondées sur un imaginaire découplé de la factualité des rapports sociaux, des mécanismes économiques et des relations internationales. 